\title{
Electrophysiologic alterations in the excitability of the sciatic and vagus nerves during early stages of sepsis
}

This article was published in the following Dove Press journal: Journal of Pain Research

\author{
Lúcio Ricardo Leite Diniz' \\ Viviane Gomes Portella ${ }^{2}$ \\ Kerly Shamira da Silva \\ Alves ${ }^{2}$ \\ Pâmella Cristina da Costa \\ Araújo \\ Ricardo Luiz Cavalcanti de \\ Albuquerque Júnior ${ }^{3}$ \\ Aline Alice Cavalcante de \\ Albuquerque ${ }^{2}$ \\ Andrelina Noronha \\ Coelho-de-Souza ${ }^{2}$ \\ José Henrique \\ Leal-Cardoso ${ }^{2}$ \\ 'Department of Physiology, Federal \\ University of Sergipe, São Cristóvão, \\ Brazil; 'Department of Physiology, \\ Superior Institute of Biomedical \\ Sciences, State University of Ceará, \\ Fortaleza, Brazil; ' ${ }^{2}$ Laboratory of \\ Morphology and Structural Biology, \\ Science and Technology Institute - \\ ITP, Aracaju, Brazil
}

Correspondence: Lúcio Ricardo Leite Diniz

Department of Physiology, Universidade Federal de Sergipe (UFS), São Cristovão, 49100-000 SE, Brazil

Tel +55 7921056644

Email luciodiniz@yahoo.com.br
Background: Nonspecific and delayed diagnosis of neurologic damage contributes to the development of neuropathies in patients with severe sepsis. The present study assessed the electrophysiologic parameters related to the excitability and conductibility of sciatic and vagus nerves during early stages of sepsis.

Materials and methods: Twenty-four hours after sepsis induced by cecal ligation and puncture (CLP) model, sciatic and vagus nerves of septic (CLP group) and control (sham group) rats were removed, and selected electric stimulations were applied to measure the parameters of the first and second components of the compound action potential. The first component originated from fibers with motor and sensory functions (Types $\mathrm{A} \alpha$ and $\mathrm{A} \beta$ fibers) with a large conduction velocity $(70-120 \mathrm{~m} / \mathrm{s})$, and the second component originated from fibers (Type A $\gamma$ ) with sensorial function. To evaluate the presence of sensorial alterations, the sensitivity to non-noxious mechanical stimuli was measured by using the von Frey test. Hematoxylin and eosin staining of the nerves was performed.

Results: We observed an increase of rheobase followed by a decrease in the first component amplitude and a higher paw withdrawal threshold in response to the application of von Frey filaments in sciatic nerves from the CLP group compared to the sham group. Differently, a decrease in rheobase and an increase in the first component amplitude of vagal $\mathrm{C}$ fibers from CLP group were registered. No significant morphologic alteration was observed.

Conclusion: Our data showed that the electrophysiologic alterations in peripheral nerves vary with the fiber type and might be identified in the first $24 \mathrm{~h}$ of sepsis, before clinical signs of neuromuscular disorders.

Keywords: sepsis, sciatic nerve, vagus nerve, neuronal excitability, neuropathy, peripheral nerves

\section{Introduction}

Despite the advances in the management of sepsis and its clinical complications, neuromuscular disorders are increasingly recognized in critically ill patients with sepsis. ${ }^{1,2}$ Several neuromuscular disorders, such as critical illness myopathy and polyneuropathy, have been found in up to $70 \%$ of critically ill patients with severe sepsis in intensive care units (ICUs) worldwide. ${ }^{3,4,28}$ Neurologic disturbances are associated with sensory defects and limb weakness, which contribute to an increase in mechanical ventilator dependence and length of hospital stay for ICU patients..$^{5-7}$ Moreover, neurologic disorders prolong physical rehabilitation and cause chronic disability in patients who survive a severe sepsis episode. ${ }^{8,9}$ Preliminary findings have suggested that, rather than degeneration of axons, the reduction in the excitability of motor and sensory fibers is due to a hyperpolarized shift in the voltage dependence of sodium caused by 
inactivation of sodium channels in peripheral nerves during the acute phase of critical illness. ${ }^{10}$

CIP attacks numerous nerves in the body, affecting more than one nerve group (e.g., sensory, motor, and vasomotor fibers) simultaneously. ${ }^{11,12}$ Many clinical manifestations of neuromuscular disorders induced by sepsis are closely linked to the impairment of sciatic and vagus nerves. ${ }^{13,14}$ Sciatic nerves are composed of myelinated fibers with large conduction velocity (Type A fibers), whereas vagus nerves are composed of myelinated Type A fibers and unmyelinated with low-conduction velocity fibers (Type $\mathrm{C}$ fibers). ${ }^{13,14}$ Previous studies have reported that vagus nerve disturbances appear to play a critical role in the immune response, especially in controlling intestinal immune activation, while sciatic nerve damages are often associated with the low back weakness, pain, tingling, and numbness found in critically ill patients. ${ }^{15-17}$

The adequate registers of initial alterations in the nerve excitability during early periods of sepsis have been limited by the use of conventional diagnostic techniques commonly used in the ICU setting that provide nonspecific results and fail to detect discrete abnormalities in the impaired nervous fiber excitability. ${ }^{18-20}$ Some electrophysiologic tests have been recognized as appropriate methodologies to investigate initial alterations of the neurologic excitability, including the register of compound motor action potential components of peripheral motor and sensitive nerves. ${ }^{21,22}$ Neurophysiologic investigation of nerves in situ by measurement of compound action potentials (CAPs) can provide relevant information about neural excitability, such as the number of active fibers and the propagation velocity of action potentials. ${ }^{23,24}$

Although previous studies provide evidences of the important rules of sciatic and vagus nerve disorders in the pathogenesis and progression of sepsis and its clinical complications, the differences in the electrophysiologic alterations found in the early period of sepsis have not yet been simultaneously evaluated and compared. Therefore, we have investigated alterations in basic neuronal properties, including electrophysiologic excitability and CAP parameters, in isolated sciatic and vagus nerves of rats within the first $24 \mathrm{~h}$ of polymicrobial sepsis (cecal ligation and puncture [CLP] model of sepsis).

\section{Materials and methods} Animals

Male Wistar rats (240-300 g) were obtained from the bioscience unit of our institution and were housed in standard conditions with free access to standard chow and tap water.
All animals used in the current study $(\mathrm{n}=60)$ were kept at a room temperature $\left(22^{\circ} \mathrm{C} \pm 2^{\circ} \mathrm{C}\right)$ with a light/dark cycle of $12 / 12 \mathrm{~h}$. All procedures described had prior approval from the animal ethics committee of the State University of Ceará $\left(n^{\circ} 07227619-3\right)$. The experiments performed followed the Office of Laboratory Animal Welfare guidelines and PHS Policy on Humane Care and Use of Laboratory Animals. All chemicals were purchased from Sigma-Aldrich (St. Louis, MO, USA).

\section{Experimental polymicrobial model of sepsis}

Sepsis was induced using the CLP model of polymicrobial sepsis as previously described. ${ }^{25}$ Animals were randomized to a sham-operated group (control group/n=20) or a CLPoperated group (septic group $/ \mathrm{n}=40$ ). Briefly, the animals were anesthetized with intraperitoneal injection of ketamine $(15 \mathrm{mg} / \mathrm{kg})$ and xylazine $(7.5 \mathrm{mg} / \mathrm{kg})$. After anesthesia, a $3 \mathrm{~cm}$ midline incision was made in the anterior abdomen and the cecum was exposed. Fecal content was dragged to the cecum ligation and held below the ileocecal junction pole with a 5/0 Prolene thread (Ethicon), without causing bowel obstruction. The cecum was punctured 10 times with an 18-gauge needle and slightly compressed until a small drop of stool appeared. The cecum was repositioned in the abdomen, and the incisions in the peritoneal wall and skin were closed. Sham-operated animals underwent an identical laparotomy, but without cecal puncture, and served as controls. All animals received $2 \mathrm{~mL}$ of saline subcutaneously immediately after surgery.

\section{Measurement of blood and peritoneum bacterial load}

To determine the bacterial load in the blood, blood samples were collected from the tail vein of sham and CLP rats at 0 and $24 \mathrm{~h}$ after surgical procedure for the induction of sepsis. Thereafter, $100 \mu \mathrm{L}$ of blood was plated on Muller-Hinton (Himedia, Mumbai, India) in accordance with the procedures supplied by the manufacturer. To measure the peritoneum bacterial load, the peritoneal cavity was previously lavaged with $5 \mathrm{~mL}$ sterile saline $(0.9 \% \mathrm{NaCl}$ solution $)$ and $100 \mu \mathrm{L}$ peritoneum fluid was collected. Shortly afterward, dilutions of 1:1000, 1:10000, and 1:100000 were made with sterile saline. One hundred microliters of each dilution was then plated on Muller-Hinton. Both blood and peritoneum samples were incubated at $37^{\circ} \mathrm{C}$ for $24 \mathrm{~h}$ under aerobic conditions. Results were expressed as colony formation units (CFUs) per milliliter counted. 


\section{Measurement of mean arterial pressure and heart rate}

All animals were submitted to 4 days of adaptation to plethysmometer conditions (adaptation period). In the end of the fourth day of adaptation and $24 \mathrm{~h}$ after sham and CLP surgeries, mean arterial pressure (MAP) and heart rate (HR) were measured by means of tail-cuff plethysmography (LE 5001; PanLab Instruments, Barcelona, Spain). The MAP and HR signals were recorded with computer software (RS232; SeDaCom software).

\section{Register of evoked CAP}

Twenty-four hours after sepsis induction, all animals were anesthetized with intraperitoneal injection of ketamine (15 $\mathrm{mg} / \mathrm{kg})$ and xylazine $(7.5 \mathrm{mg} / \mathrm{kg})$ and killed by $\mathrm{CO}_{2}$ asphyxiation. Shortly after, their sciatic and vagus nerves were removed and placed in a moist chamber filled with fresh modified Locke's solution (mmol/L): $140 \mathrm{NaCl}, 5.6 \mathrm{KCl}, 2.2$ $\mathrm{CaCl}_{2}, 1.2 \mathrm{MgCl}_{2}, 10$ glucose, and 10 Tris-(hydroxymethylaminomethane), and $\mathrm{pH}$ 7.4.

Nerves then underwent a stabilization period varying from $30 \mathrm{~min}$ (vagus) to $2 \mathrm{~h}$ (sciatic) until the stable peakto-peak CAP amplitude was achieved. Sciatic nerve CAPs were obtained through the application of repetitive stimuli at a frequency of $0.2 \mathrm{~Hz}$, with $40 \mathrm{~V}$ electrical pulses at 100 $\mu$ s durations in 6-8 $\mathrm{mm}$ segments of nerves. Vagus nerves were stimulated at a frequency of $0.2 \mathrm{~Hz}$, with $60 \mathrm{~V}$ electrical pulses of $1,500 \mu \mathrm{s}$ duration in $4-8 \mathrm{~mm}$ segments. The electrical stimulus was delivered by a stimulus isolation unit (Model SIU4678; Grass Instrument, Quincy, MA, USA) connected to a stimulator (Model S48; Grass Instrument). CAP was recorded with platinum electrodes placed 40-60 $\mathrm{mm}$ from stimulating electrodes and continuously monitored using an oscilloscope (model 547; Tektronix, Inc., Portland, OR, USA). Data were recorded and analyzed with computer acquisition hardware (Digidata 1440A model; Molecular Devices, Sunnyvale, CA, USA) and Clampex software (Molecular Devices). ${ }^{26}$

The parameters measured in evoked CAP were peak positive amplitude (from baseline) and conduction velocity of CAP components. Rheobase and chronaxie, parameters related to excitability, were determined from strengthduration curves generated by the stimulation with voltage square waves. Rheobase was defined as the threshold stimulus voltage required for an active response with a long-duration pulse $(1000 \mu \mathrm{s})$. Chronaxie is the threshold duration for an active response with a stimulus at twice the rheobase voltage. ${ }^{27}$

\section{Mechanical sensitivity test}

Animals' sensitivity to non-noxious mechanical stimuli was measured using von Frey hairs/Semmes-Weinstein monofilaments (Stoelting Co., Wood Dale, IL, USA). Twenty-four hours after operation, mechanical stimulation sensitivity was assessed. Tests were administered by recording paw withdrawal response to a serially increasing force on a von Frey filament. Initially, the animals were acclimatized to a raised platform for $1 \mathrm{~h}$ each day 3 days before testing and an hour just before testing. Filaments varying from 1.0 to $6.5 \mathrm{~g}$ were applied to the plantar surface of the hind paw, pressed until it bent, and kept in place for $8 \mathrm{sec}$. At $60 \mathrm{sec}$ intervals, application with the same filament was repeated, and the response was recorded only when six consecutive positive responses (withdrawal of the paw off the floor after application) for a single filament were observed. The withdrawal threshold, the stimulus intensity at which the paw withdrawal is detected $50 \%$ of the time, was calculated and compared between experimental groups. ${ }^{26,29}$

\section{Histologic analysis}

Sciatic and vagus nerves were removed immediately after cervical dislocation. The specimens were fixed in $10 \%$ neutral buffered formalin solution, washed in water, dehydrated in a graded ethanol series, cleared in xylene, embedded in paraffin, and cut into 5- $\mu \mathrm{m}$-thick cross or longitudinal sections. The longitudinal and cross paraffin sections were stained with hematoxylin and eosin (H\&E), and examined using light microscopy.

\section{Statistical analysis}

Data are expressed as the mean \pm standard error of the mean. Statistical analyses were conducted with GraphPadPrism ${ }^{\circledR}$ 5.0 software (GraphPad Software Inc, La Jolla, CA, USA). Statistical significance was assessed using Student's $t$-test and one-way analysis of variance followed by Newman-Keuls multiple-comparison test. In all cases, $P<0.05$ was considered statistically significant

\section{Results \\ Survival curve}

Figure 1 illustrates the survival rate of sham and CLP groups within $120 \mathrm{~h}$ after induction of sepsis. No deaths occurred in the sham-operated animals. In CLP-operated rats, we observed about $20 \%$ and $30 \%$ mortality during 24 and $48 \mathrm{~h}$ after CLP, respectively. 


\section{Bacterial growth, MAP, and HR}

Table 1 summarizes the MAP, HR, and bacterial growth of blood and peritoneal fluid culture isolated from sham- and CLP-operated rats. Approximately $90 \%$ of CLP-operated rat samples showed a bacterial load in the order of $10^{4}$ and $10^{6}$ CFU per $\mathrm{mL}$ of the blood and peritoneum fluid, respectively. No bacterial growth was detected in growing bacteria cultures

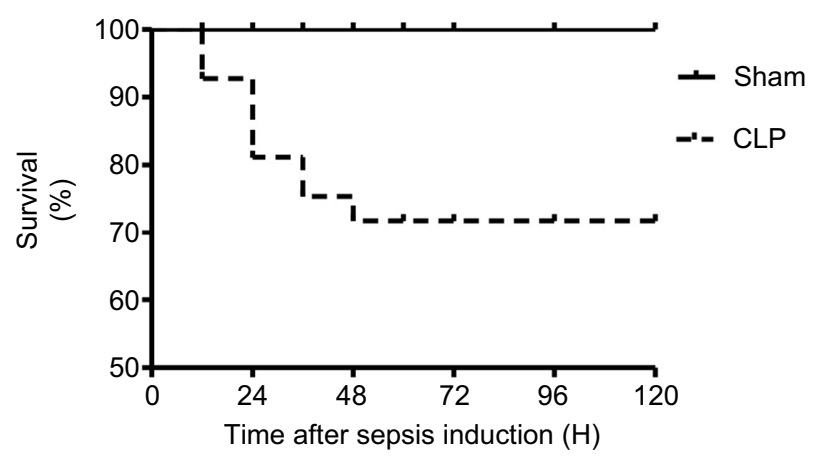

Figure I Survival rate of sham- and CLP-operated rats observed for $120 \mathrm{~h}$ postsurgery.

Notes: The survival rate was analyzed using log-rank test. $n=20 /$ each group. CLPoperated rats showed from $20 \%$ to $30 \%$ mortality between 24 and $48 \mathrm{~h}$ post-CLP, compared to $0 \%$ mortality observed in sham-operated rats.

Abbreviation: CLP, cecal ligation and puncture. of the blood and peritoneal fluid from sham-operated rats. In addition, CLP group showed a great reduction in MAP (from $102.31 \pm 2.32$ to $75.70 \pm 9.53 \mathrm{mmHg}, 0$ and $24 \mathrm{~h}$, respectively) followed by increasing HR (from $363.06 \pm 9.12$ to $476.34 \pm 33.68 \mathrm{bpm}, 0$ and $24 \mathrm{~h}$, respectively). No significant alterations in the MAP and HR obtained from sham-operated rats were detected during the periods evaluated.

\section{Neuronal excitability}

Rheobase (Figure 2A) and chronaxie values (Figure 2B) are the important parameters directly related to neuronal excitability. Both parameters were measured from sciatic and vagus nerves of experimental groups $24 \mathrm{~h}$ following surgery. In sciatic nerves, a significant increase $(P<0.05)$ was observed in the rheobase values of CLP-operated rats $(3.49 \pm 0.07 \mathrm{~V})$ compared to sham-operated rats $(3.09 \pm 0.05 \mathrm{~V})$. In contrast, a significant decrease in rheobase values was found in vagus nerves of CLP-operated animals $(21.00 \pm 1.87 \mathrm{~V})$ compared to the sham group $(27.22 \pm 2.33$ V). No significant difference was observed in chronaxie values between sham and CLP groups in our evoked CAP register of sciatic and vagus nerves.

Table I MAP, HR, and BBL and PBL cultures isolated from sham- and CLP-operated rats 0 and $24 \mathrm{~h}$ after sepsis induction

\begin{tabular}{llllll}
\hline Time (h) & & BBL (CFU- $\left.\log _{10} / \mathbf{m L}\right)$ & PBL $\left(\right.$ CFU- $\log _{10} /$ cavity) & MAP (mmHg) & HR (bpm) \\
\hline 0 & Sham $(n=6)$ & - & - & $98.82 \pm 2.56$ & $349.22 \pm 9.41$ \\
& CLP $(n=9)$ & - & - & $102.31 \pm 2.32$ & $363.06 \pm 9.12$ \\
24 & Sham $(\mathrm{n}=6)$ & 0 & 0 & $109.12 \pm 2.35$ & $340.79 \pm 5.58$ \\
& CLP $(\mathrm{n}=6)$ & $4.15 \pm 0.73^{*}$ & $6.42 \pm 0.24^{*}$ & $75.70 \pm 9.53^{*}$ & $476.34 \pm 33.68^{*}$ \\
\hline
\end{tabular}

Notes: *Indicates a significant difference related to 0 and $24 \mathrm{~h}$ after sepsis induction $(P<0.05$, Student's $t$-test).

Abbreviations: BBL, blood bacterial load; CFU, colony formation unit; CLP, cecal ligation and puncture; HR, heart rate; MAP, mean arterial pressure; PBL, peritoneum bacterial load.

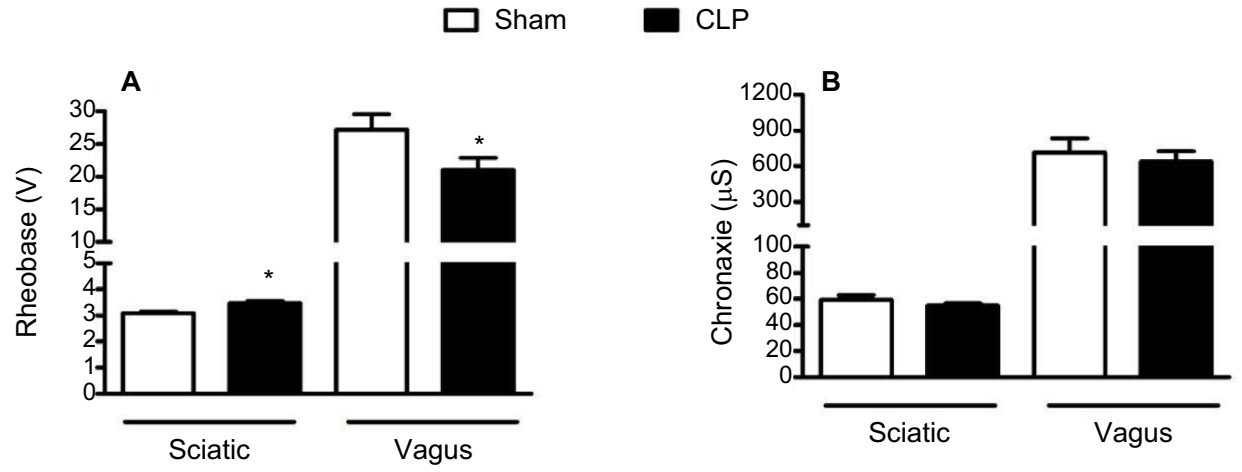

Figure 2 Register of evoked CAP of parameters related to the excitability of peripheral nerves.

Notes: Rheobase (A) and chronaxie (B) measured on evoked CAP register of sciatic and vagus nerves isolated from sham-operated rats ( $\mathrm{n}=10$ and 8 , respectively) and CLP-operated rats ( $n=10$ and 9, respectively). *Indicates a significant difference related to sham-operated rats $(P<0.05$, Student's $t$-test).

Abbreviations: CAP, compound action potential; CLP, cecal ligation and puncture. 
The values for amplitude and conducting velocity parameters are shown in Figure 3. No alterations were observed in the conducting velocity of the sciatic or vagus nerves. However, the first component amplitude of the CAP was lower in sciatic nerves of CLP-operated rats $(6.60 \pm 0.29 \mathrm{mV})$ than in shamoperated rats $(8.70 \pm 0.93 \mathrm{mV})$. There was also a significant increase in CAP amplitude of vagal $\mathrm{C}$ fibers in the CLP group $(1.33 \pm 0.33 \mathrm{mV})$ compared to the sham group $(0.54 \pm 0.05 \mathrm{mV})$.

\section{Mechanical sensitivity}

The electronic von Frey test showed a decrease in mechanical sensory sensitivity; paw withdrawal threshold was greater in the CLP group (EC50=20.05 $\pm 2.8 \mathrm{~g}$ ) than in sham-operated rats $(\mathrm{EC} 50=15.11 \pm 1.76 \mathrm{~g})$, as seen in Figure 4. Additionally, a downward and a rightward shift were observed in the paw withdrawal curve of septic animals compared to the respective curve of control animals.
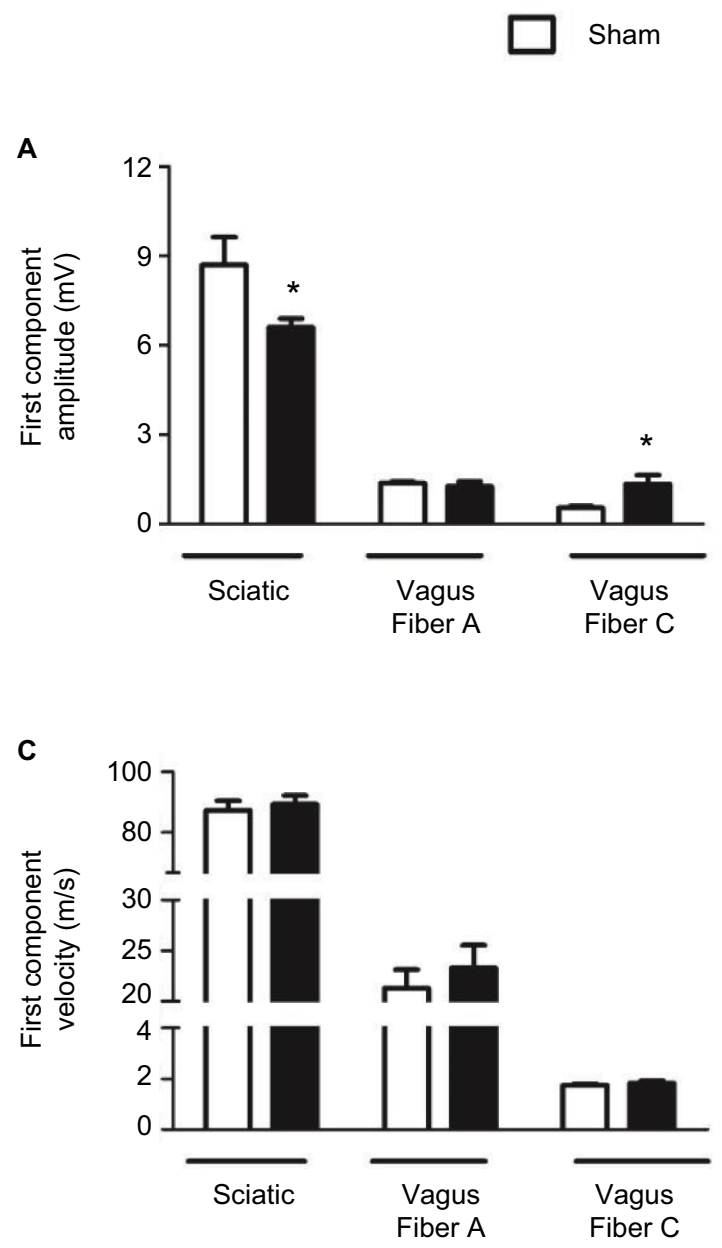

\section{Morphologic analysis}

At the first $24 \mathrm{~h}$ of sepsis, neither the vagus nerves nor the sciatic nerves of septic animals (CLP group) showed significant morphologic changes compared with the sham group. H\&E staining demonstrated similar histologic structures in transversal sections of vagus and sciatic nerves of both experimental groups, as illustrated in Figure 5.

\section{Discussion}

The present study has investigated the alterations in the excitability of isolated vagus and sciatic nerves using electrically evoked CAP, especially parameters associated with neuronal excitability (e.g., chronaxie and rheobase), during the early stages of polymicrobial model (CLP model). In general, we observed a qualitative alteration in neuronal excitability, which differed greatly between the sciatic nerve, where it decreased, and the vagus nerve, where it increased.

CLP
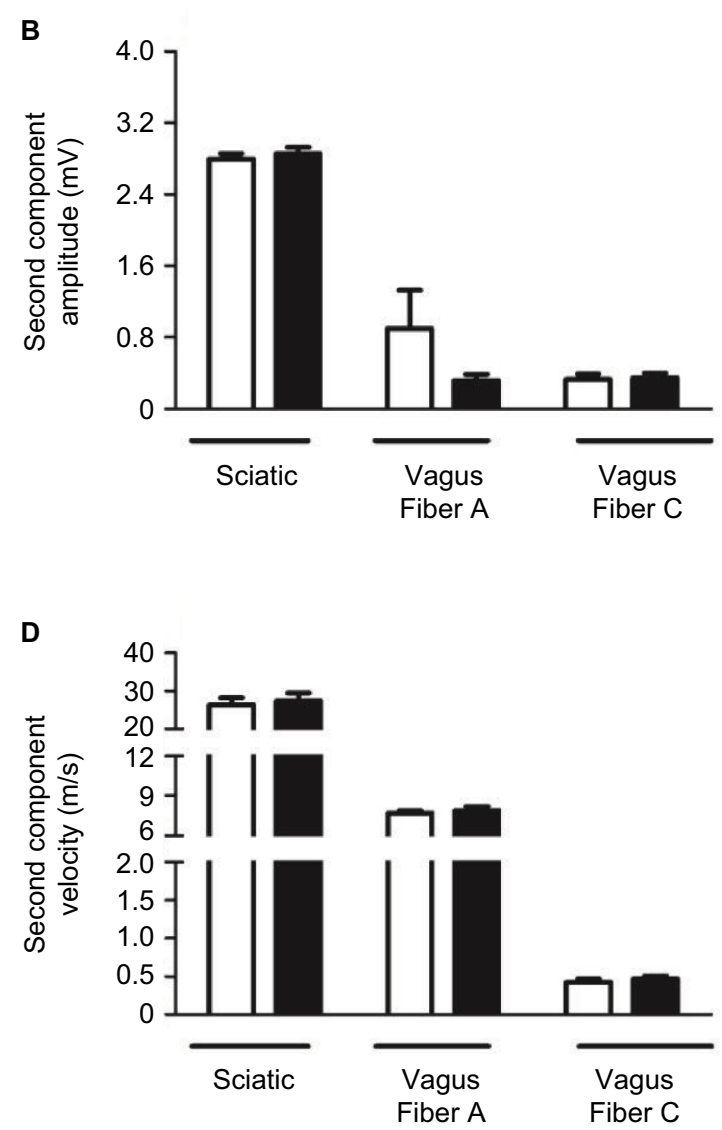

Figure 3 Register of parameters of evoked CAP related to the conductibility of peripheral nerves.

Notes: Amplitude of the first (A) and second (B) components in sham-operated $(n=8)$ and CLP-operated rats ( $n=9)$. Conduction velocity of the first (C) and second (D) CAP components in sham-operated $(n=10)$ and CLP-operated rats $(n=8)$ measured in registers of evoked CAPs of sciatic and vagus nerves $24 \mathrm{~h}$ after sepsis induction. *Indicates a significant difference related to sham-operated rats $(P<0.05$, Student's $t$-test).

Abbreviations: CAP, compound action potential; CLP, cecal ligation and puncture. 
The presence of bacteria in the peritoneum fluid and the bloodstream (bacteremia) added to hypotension (Table 1) was measured in the laboratory, and it was the clinical sign used to characterize the sepsis induction, whereas the CLP-operated rats that showed no bacterial growth in growing bacteria cultures of blood and hypotension were discarded. The mortality rate of CLP-operated rats was $\sim 30 \%$, comparable to mortality among ICU patients with severe sepsis in diverse countries around the world. ${ }^{27}$ These data provide evidences that CLPoperated rats developed sepsis with similar clinical signs and degree of severity to those observed in clinics.

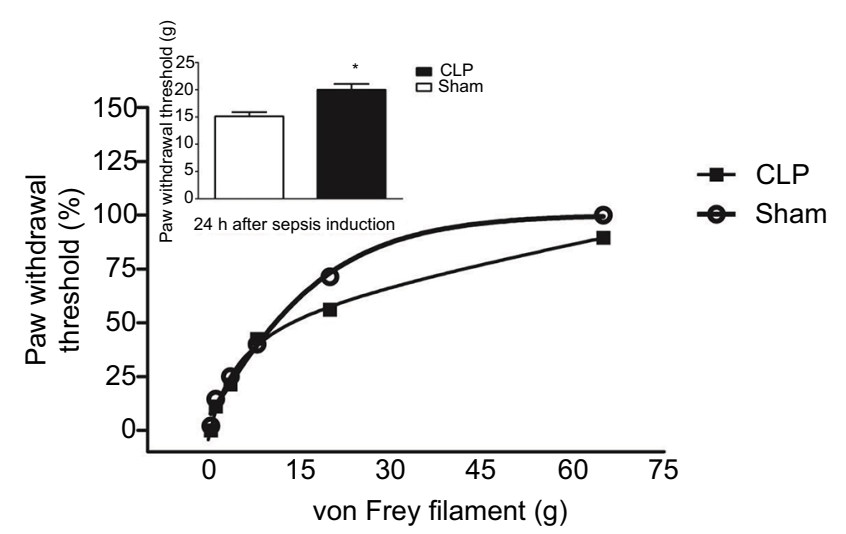

Figure 4 Measurements of mechanical sensibility.

Notes: Von Frey test shows decreased sensitivity to mechanical stimulation in the paws of CLP-operated rats as evidenced by a downward and a rightward shift in the paw withdrawal curve of CLP-operated rats compared to sham-operated $(n=10 /$ each group) ones. Inset shows the paw withdrawal threshold observed in $50 \%$ of animals of both experimental groups.

Abbreviation: CLP, cecal ligation and puncture.
Sensory defects and limb weakness, caused by primary axonal degeneration in peripheral motor and sensitive fibers, are clinical complications frequently found in ICU patients with severe sepsis. ${ }^{1-8}$ Nonspecific and delayed diagnosis of neuromuscular disorders have contributed to the high incidence of sepsis-induced neuropathies. ${ }^{19,20}$ This is aggravated by the fact that septic patients are generally bedridden, which can mask the subtle onset of neurologic symptoms. ${ }^{8,9}$ Functional investigation of nerves in situ may provide relevant scientific and clinical information through the measurement of alterations in nerve excitability, such as onset time and magnitude of neurologic alterations. The results reported here may contribute to the early diagnosis and better understanding of the cellular mechanisms involved in neuromuscular disorders caused by sepsis, such as CIP. ${ }^{20-24}$

Considering that CIP is a neurologic disorder that often affects sensory, motor, and vasomotor nerve fibers, the selection of the two peripheral nerves used in this study followed the criteria of presenting different morphology and physiologic functions..$^{29-33}$ These nerves were chosen to evaluate the effect of sepsis on the excitability of a somatic (sciatic) and autonomic (vagus) nerves. ${ }^{30-32}$ In the sciatic nerve, we measured parameters of the first and second CAP components in myelinated fibers. ${ }^{33}$ The first component originated from fibers with motor and sensory functions (Types $\mathrm{A} a$ and $\mathrm{A} \beta$ fibers) with a large conduction velocity $(70-120 \mathrm{~m} / \mathrm{s})$. The second component originated from fibers (Type A $\gamma$ ) with sensorial functions, conducting sensations of touch and pressure that have a lower
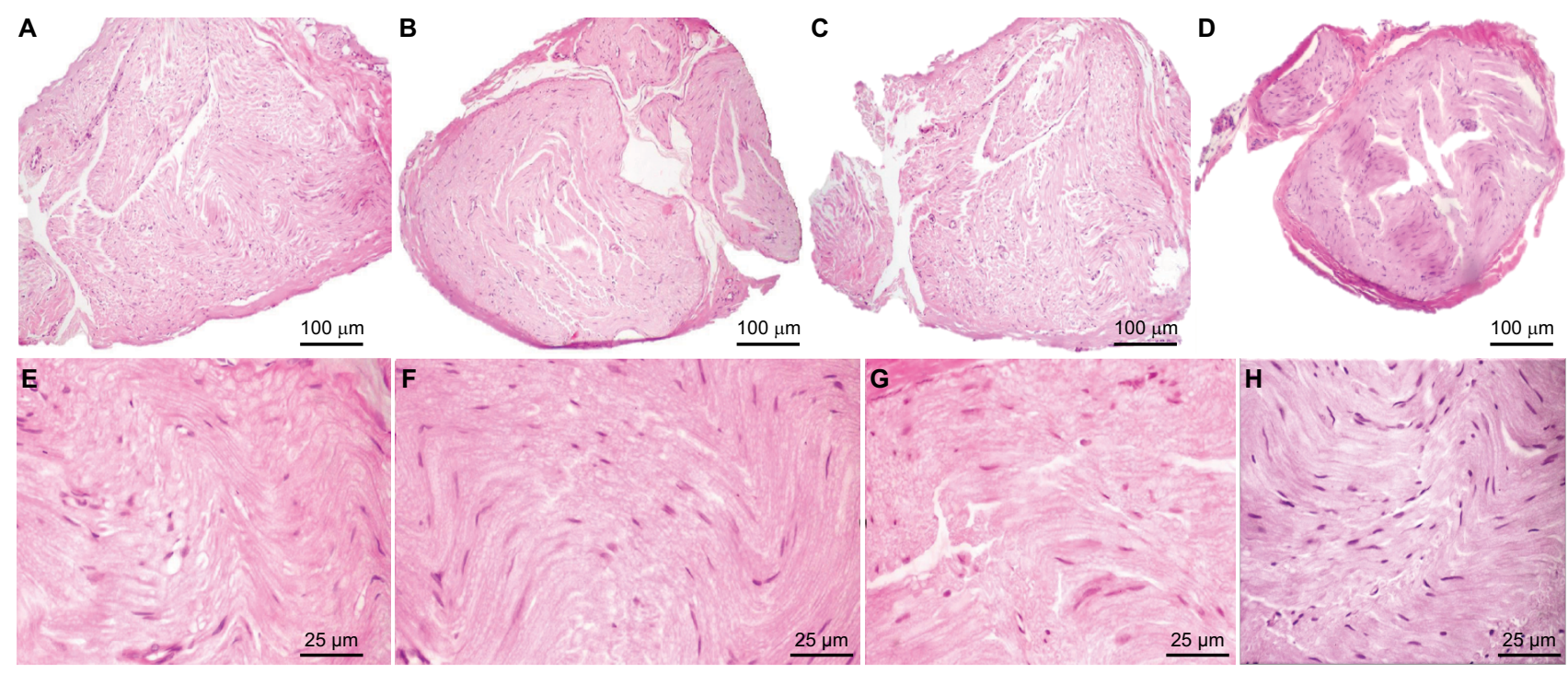

Figure 5 Transversal sections with H\&E staining of sciatic and vagus nerves $24 \mathrm{~h}$ after operation in the sham and CLP groups.

Notes: Sciatic structure of sham-operated rats by H\&E staining, scale bars $=100 \mu \mathrm{m}$ (A) and $25 \mu \mathrm{m}$. (E); sciatic structure of CLP-operated rats by H\&E staining, scale bars=100 $\mu \mathrm{m}$ (B) and $25 \mu \mathrm{m}$ (F); vagus structure of sham-operated rats by H\&E staining, scale bars=100 $\mu \mathrm{m}$ (C) and $25 \mu \mathrm{m}$ (G); vagus structure of CLP-operated rats by H\&E staining, scale bars $=100 \mu \mathrm{m}$ (D) and $25 \mu \mathrm{m}(\mathbf{H})$.

Abbreviations: CLP, cecal ligation and puncture; H\&E, hematoxylin and eosin. 
conduction velocity $(30-70 \mathrm{~m} / \mathrm{s})$ than does Type A $a \cdot{ }^{21-24} \mathrm{In}$ contrast, the parasympathetic vagus nerve is composed of Type A (myelinated, conduction velocity [4-120 m/s]) and Type C fibers (unmyelinated, conduction velocity $[<2 \mathrm{~m} / \mathrm{s}]$ ). ${ }^{29-31}$ Thus, the nerves and components selected for the measurement represent a large range of fiber types and physiologic functions.

Based on CAP evocation measurements, we observed an increase in rheobase (Figure 2A) followed by a decrease in the first component amplitude of sciatic nerves from CLPoperated rats compared to sham-operated rats (Figure 3A). These data suggest that alterations might eventually progress to motor neuron damage starting with reduced excitability. These changes might be detected in sciatic nerves during the early phase of sepsis, before the appearance of clinical signs of gross derangement in motor neurons (e.g., weakness, paresthesia, and hyperesthesia) found in the late phases of sepsis. ${ }^{35,36}$ We observed a higher paw withdrawal threshold in response to the application of increasing von Frey filaments in CLP-operated rats compared to sham-operated rats (Figure 4 ), indicating a reduction in the sensitivity to mechanical stimulation induced by sepsis $\mathrm{s}^{20-24}$ and corroborating with our evoked CAP registers.

In contrast to sciatic nerves, we observed a decrease in rheobase (Figure 2A) followed by an increase in the first component amplitude in vagal $\mathrm{C}$ fibers from CLP-operated rats compared to sham-operated rats (Figure 3A), indicating an increase in vagal excitability. ${ }^{29,30}$ Our results are consistent with the previous studies that have proposed that an increase in vagal activity modulates immune dysregulation and motility of visceral organs, in addition to its involvement in important sensory alterations such as hyperalgesia, allodynia, and pain in the polymicrobial sepsis. ${ }^{12-17}$ It is noteworthy that CAP amplitude alterations were detected in our registers within the first $24 \mathrm{~h}$ of sepsis, while these neuronal changes are not commonly observed before the first week of sepsis by clinical electromyography measures and record signals originated from muscle contraction in response to neural stimulation used in clinics. ${ }^{34-36}$

We also observed that the alterations registered in neurologic excitability of vagus and sciatic nerves seem to precede morphologic changes, as suggested by the absence of the important histologic alterations in HE-stained transversal sections of both peripheral nerves of CLP-operated rats compared with sham-operated rats (Figure 5).

Although CLP and LPS models of sepsis are recognized as experimental models with differences in kinetics and magnitude of inflammatory response, as a consequence leading to several conflicting results in literature, ${ }^{37}$ our data are in agree- ment with recent study published by Miura et al, ${ }^{39}$ in which the authors showed that sciatic nerve conduction abnormalities precede morphologic alterations after $48 \mathrm{~h}$ of LPS. ${ }^{38}$

Taken together, our results are important to show not only that peripheral nerve excitability abnormalities depend on the type of nervous fiber, but also that they might be detected prior to morphologic changes and usual clinical tests to register neuromuscular disorders.

\section{Conclusion}

In summary, this is the first report documenting sepsisinduced in situ alterations in electrophysiologic parameters of neurologic excitability in two peripheral nerves with important physiologic functions..$^{30,32}$ Under severe septic conditions, functional alterations of these nerves were already detected: excitability of sciatic nerves is reduced, whereas vagal excitability is increased in the first $24 \mathrm{~h}$ of sepsis. Twenty-four hours after the onset of sepsis, no clinical manifestations of neuromuscular disorders are detected with the clinical tests in use. Our findings have clinical relevance because they describe the early onset of the important possible neural alterations present but still subclinical in human sepsis. Therefore, our results contribute to a better understanding of the pathophysiology of this disease.

\section{Acknowledgments}

The authors are grateful to the staff members of the Institute of Technology and Research for their technical assistance.

\section{Author contributions}

All authors contributed toward data analysis, drafting and revising the paper and agree to be accountable for all aspects of the work.

\section{Disclosure}

The authors report no conflicts of interest in this work.

\section{References}

1. Bolton CF. Impact commentaries. Polyneuropathy in critically ill patients. J Neurol Neurosurg Psychiatry. 2012;83(5):2779-2782.

2. Takei T. [Intensive care unit-acquired weakness: development of polyneuropathy and myopathy in critically ill patients]. Brain Nerve. 2014;66(2):161-170. Japanese.

3. Johnson KL. Neuromuscular complications in the intensive care unit: critical illness polyneuromyopathy. AACN Adv Crit Care. 2007; 18(2):167-180; quiz 181-162.

4. Koshy K, Zochodne DW. Neuromuscular complications of critical illness. Handb Clin Neurol. 2013;115:759-780.

5. Wang XK, Zhu J, Zhang HL. Critical illness polyneuropathy and myopathy are common neuromuscular complications secondary to sepsis. Neurol Sci. 2013;34(1):129-130. 
6. Eikermann M, Koch G, Gerwig M, et al. Muscle force and fatigue in patients with sepsis and multiorgan failure. Intensive Care Med. 2006;32(2):251-259.

7. Garnacho-Montero J, Madrazo-Osuna J, Garcia-Garmendia JL, et al. Critical illness polyneuropathy: risk factors and clinical consequences. A cohort study in septic patients. Intensive Care Med. 2001;27(8): 1288-1296.

8. Ehlenbach WJ, Larson EB, Curtis JR, Hough CL. Physical function and disability after acute care and critical illness hospitalizations in a prospective cohort of older adults. J Am Geriatr Soc. 2015;63(10): 2061-2069.

9. Herridge M, Cameron JI. Disability after critical illness. N Engl J Med. 2013;369(14):1367-1369.

10. Koch S, Bierbrauer J, Haas K, et al. Critical illness polyneuropathy in ICU patients is related to reduced motor nerve excitability caused by reduced sodium permeability. Intensive Care Med Exp. 2016;4(1):10.

11. Witt NJ, Zochodne DW, Bolton CF, et al. Peripheral nerve function in sepsis and multiple organ failure. Chest. 1991;99(1):176-184.

12. Friedrich O. Critical illness myopathy: sepsis-mediated failure of the peripheral nervous system. Eur J Anaesthesiol. Suppl. 2008;42:73-82.

13. Feinberg J, Sethi S. Sciatic neuropathy: case report and discussion of the literature on postoperative sciatic neuropathy and sciatic nerve tumors. HSS J. 2006;2(2):181-187.

14. Borovikova LV, Ivanova $\mathrm{S}$, Zhang $\mathrm{M}$, et al. Vagus nerve stimulation attenuates the systemic inflammatory response to endotoxin. Nature. 2000;405(6785):458-462.

15. Axer H, Grimm A, Pausch C, et al. The impairment of small nerve fibers in severe sepsis and septic shock. Crit Care. 2016;20:64.

16. Latronico N, Filosto M, Fagoni N, et al. Small nerve fiber pathology in critical illness. PLoS One. 2013;8:e75696.

17. Matteoli G, Boeckxstaens GE. The vagal innervation of the gut and immune homeostasis. Gut. 2013;62(8):1214-1222.

18. Lefaucheur JP, Nordine T, Rodriguez P, Brochard L. Origin of ICU acquired paresis determined by direct muscle stimulation. J Neurol Neurosurg Psychiatry. 2006;77(4):500-506.

19. Bird SJ. Diagnosis and management of critical illness polyneuropathy and critical illness myopathy. Curr Treat Options Neurol. 2007;9(2): 85-92.

20. Chawla J, Gruener G. Management of critical illness polyneuropathy and myopathy. Neurol Clin. 2010;28(4):961-977.

21. Lacomis D. Electrophysiology of neuromuscular disorders in critical illness. Muscle Nerve. 2013;47(3):452-463.

22. Latronico N, Smith M. Introducing simplified electrophysiological test of peripheral nerves and muscles in the ICU: choosing wisely. Intensive Care Med. 2014;40:746-748.

23. Trojaborg W. Electrophysiologic techniques in critical illness-associated weakness. J Neurol Sci. 2006;242:83-85.
24. Spivak L, Auerbach C, Vambutas A, Geshkovich S, Wexler L, Popecki B. Electrical compound action potentials recorded with automated neural response telemetry: threshold changes as a function of time and electrode position. Ear Hear. 2011;32(1):104-113.

25. Rittirsch D, Huber-Lang MS, Flierl MA, Ward PA. Immunodesign of experimental sepsis by cecal ligation and puncture. Nat Protoc. 2009;4:31-36.

26. Ferreira-da-Silva FW, da Silva-Alves KS, Lemos-Dos-Santos M, et al. n5-STZ diabetic model develops alterations in sciatic nerve and dorsal root ganglia neurons of Wistar rats. ISRN Endocrinol. 2013;2013:638028.

27. Holsheimer J, Dijkstra EA, Demeulemeester H, Nuttin B. Chronaxie calculated from current-duration and voltage-duration data. J Neurosci Methods. 2000;97(1):45-50.

28. Mayr FB, Yende S, Angus DC. Epidemiology of severe sepsis. Virulence. 2014;5(1):4-11.

29. Crone C, Krarup C. Neurophysiological approach to disorders of peripheral nerve. Handb Clin Neurol. 2013;115:81-114.

30. Axer H, Grimm A, Porzelius C, et al. Impairment of small somatic and autonomic nerve fibres in intensive care unit patients with severe sepsis and critical illness polyneuropathy - a single center controlled observational study. BMC Neurol. 2013;13:159.

31. Chang YT, Wann SR, Tsai JS, et al. The role of autonomic nervous system function in hypothermia-mediated sepsis protection. Am J Emerg Med. 2013;31(2):375-380.

32. Martin M, Benzina O, Szabo V, et al. Morphology and nanomechanics of sensory neurons growth cones following peripheral nerve injury. PLoS One. 2013;8(2):e56286.

33. Gogiashvili L, Abashidze T, Tsagareli Z, Dgebuadze M, Kvachadze T. [Functional morphology of the vagus nerve nuclei changes in the medulla oblongata (N. Ambiguus, N. Dorsalis), induced by influenza a $(\mathrm{H}(3) \mathrm{N}(\mathrm{I})$ ) in experiment]. Georgian Med News. 2012(213):55-62. Russian.

34. Wang XK, Zhang HL, Zhu J. Critical illness polyneuropathy/critical illness myopathy and acute motor-sensory axonal neuropathy. J Neurosurg Spine. 2013;18(4):416-418.

35. Rannou F, Pennec JP, Rossignol B, et al. Effects of chronic sepsis on rat motor units: experimental study of critical illness polyneuromyopathy. Exp Neurol. 2007;204(2):741-747.

36. Tennila A, Salmi T, Pettila V, Roine RO, Varpula T, Takkunen O. Early signs of critical illness polyneuropathy in ICU patients with systemic inflammatory response syndrome or sepsis. Intensive Care Med. 2000;26(9):1360-1363.

38. Kingsley SM, Bhat BV. Differential paradigms in animal models of sepsis. Curr Infect Dis Rep. 2016;18(9):26.

39. Miura A, Hino H, Uchida K, Inoue S, Tateda T. Peripheral nerve conduction abnormalities precede morphological alterations in an experimental rat model of sepsis. J Anesth. 2016;30(6):961-969.
Journal of Pain Research

\section{Publish your work in this journal}

The Journal of Pain Research is an international, peer reviewed, open access, online journal that welcomes laboratory and clinical findings in the fields of pain research and the prevention and management of pain. Original research, reviews, symposium reports, hypothesis formation and commentaries are all considered for publication.

\section{Dovepress}

The manuscript management system is completely online and includes a very quick and fair peer-review system, which is all easy to use. Visit http://www.dovepress.com/testimonials.php to read real quotes from published authors. 